\title{
Computing the number of realizations of a Laman graph
}

\author{
Jose Capco*†‡ Matteo Gallet ${ }^{\S \ddagger}$ Georg Grasegger ${ }^{\S}$ \\ Christoph Koutschan $\$ \ddagger \|$ Niels Lubbes $\$$ Josef Schicho*
}

Laman graphs model planar frameworks which are rigid for a general choice of distances between the vertices. There are finitely many ways, up to isometries, to realize a Laman graph in the plane. In a recent paper we provide a recursion formula for this number of realizations using ideas from algebraic and tropical geometry. Here, we present a concise summary of this result focusing on the main ideas and the combinatorial point of view.

\section{Introduction}

Suppose that we are given a graph $G$ with edges $E$. We consider the set of all possible realizations of the graph in the plane such that the lengths of the edges coincide with some edge labeling $\lambda: E \rightarrow \mathbb{R}_{\geq 0}$. Edges and vertices are allowed to overlap in such a realization. For example, suppose that $G$ has four vertices and is a complete graph minus one edge. Figure 1 shows all possible realizations of $G$ up to rotations and translations, for a given edge labeling. We say that a property holds for a general edge labeling if it holds for all edge labelings
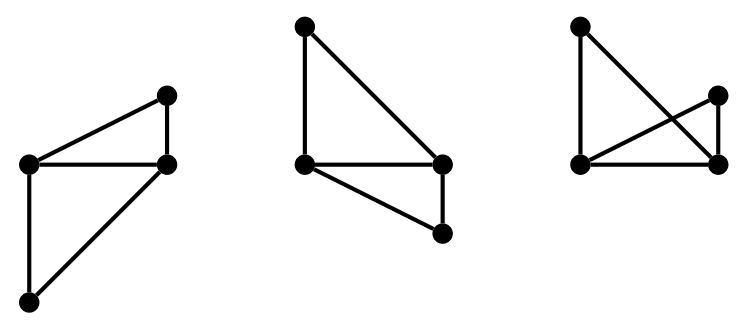

Figure 1: Realizations of a graph up to rotations and translations.

\footnotetext{
${ }^{*}$ Research Institute for Symbolic Computation (RISC), Johannes Kepler University Linz

${ }^{\dagger}$ Supported by the Austrian Science Fund (FWF): P28349

${ }^{\ddagger}$ Supported by the Austrian Science Fund (FWF): W1214-N15, project DK9

§Johann Radon Institute for Computational and Applied Mathematics (RICAM), Austrian Academy of Sciences

"Supported by the Austrian Science Fund (FWF): P26607

"Supported by the Austrian Science Fund (FWF): P29467
} 
belonging to a dense open subset of the vector space of all edge labelings. Here we address the following problem:

For a given graph determine the number of realizations up to rotations and translations for a general edge labeling.

The realizations of a graph can be considered as structures in the plane, which are constituted by rods connected by rotational joints. If a graph with an edge labeling admits infinitely (finitely) many realizations up to rotations and translations, then the corresponding planar structure is flexible (rigid).

The study of rigid structures, also called frameworks, was originally motivated by mechanics and architecture, and it goes back at least to the 19th century. Nowadays, there is still a considerable interest in rigidity theory [4] due to various applications in natural science and engineering.

A graph is called generically rigid (or isostatic) if a general edge labeling yields a rigid realization. No edge in a generically rigid graph can be removed without losing rigidity, that is why such graphs are also called minimally rigid in the literature. The complete graph on four vertices $K_{4}$ is for instance not considered to be generically rigid, since for a general choice of edge lengths it will not have a realization. Gerard Laman [6] characterized the property of generic rigidity in terms of the number of edges and vertices of the graph and its subgraphs, hence such objects are also known as Laman graphs.

Theorem 1.1. Let $G=(V, E)$ be a graph. Then $G$ is generically rigid if and only if $|E|=$ $2|V|-3$, and for every subgraph $G^{\prime}=\left(V^{\prime}, E^{\prime}\right)$ it holds $\left|E^{\prime}\right| \leq 2\left|V^{\prime}\right|-3$.

All realizations of a Laman graph can be obtained as the solution set of a system of algebraic equations, where the edge labels are interpreted as parameters. In general it is difficult to produce results on the number of real solutions of such systems. In such situations, one often switches to a complex setting; this also enables us to apply results from algebraic geometry. Hence, from now on, we are interested in the number of complex solutions, up to an equivalence relation on $\mathbb{C}^{2}$ generalizing direct isometries of $\mathbb{R}^{2}$; this number is the same for any general choice of parameters, so we call it the Laman number of the graph. For some graphs up to 8 vertices, this number had been computed using random values for the parameters [5] - this means that it is very likely, but not absolutely certain, that these computations give the true numbers. Upper and lower bounds of Laman numbers are considered in $[3,7,1]$. Note that for many Laman graphs there exists a labeling such that the number of real realizations is precisely the Laman number. However, there are Laman graphs for which the Laman number gives only an upper bound on the number of real realizations.

Our main result is a combinatorial algorithm that computes the number of complex realizations of any given Laman graph; it is much more efficient than just solving the corresponding nonlinear system of equations. The algorithm and its correctness proof are presented in detail in [2]. The purpose of the current paper is to provide a concise summary of this result focusing on the main ideas and the algorithmic point of view rather than on proofs.

\section{Main result}

By a graph we mean a finite, connected, undirected graph without self-loops or multiple edges. We write $G=(V, E)$ to denote a graph $G$ with vertices $V$ and edges $E$. An edge $e$ between two vertices $u$ and $v$ is denoted by $\{u, v\}$. 
Using nonnegative real labels, the number of embeddings is not well-defined, since it may depend on the actual labeling and not only on the graph. In order to define a number that depends only on the graph, we use a complex setting.

Definition 2.1. Let $G=(V, E)$ be a graph.

$\triangleright$ A labeling of $G$ is a function $\lambda: E \longrightarrow \mathbb{C}$. The pair $(G, \lambda)$ is called a labeled graph.

$\triangleright$ A realization of $G$ is a function $\rho: V \longrightarrow \mathbb{C}^{2}$. Let $\lambda$ be a labeling of $G$ : we say that a realization $\rho$ is compatible with $\lambda$ if for each $e \in E$ the distance between its endpoints agrees with its label:

$$
\lambda(e)=\langle\rho(u)-\rho(v), \rho(u)-\rho(v)\rangle, \quad e=\{u, v\},
$$

where $\langle x, y\rangle=x_{1} y_{1}+x_{2} y_{2}$.

A labeled graph $(G, \lambda)$ is realizable if and only if there exists a realization $\rho$ that is compatible with $\lambda$.

We say that two realizations of a graph $G$ are equivalent if and only if there exists a direct isometry $\sigma$ of $\mathbb{C}^{2}$ between them, where $\sigma$ is a map of the form

$$
\left(\begin{array}{l}
x \\
y
\end{array}\right) \longmapsto A \cdot\left(\begin{array}{l}
x \\
y
\end{array}\right)+b,
$$

where $A \in \mathbb{C}^{2 \times 2}$ is an orthogonal matrix with determinant 1 and $b \in \mathbb{C}^{2}$.

Definition 2.2. A labeled graph $(G, \lambda)$ is called rigid if it is realizable and there are only finitely many realizations compatible with $\lambda$, up to equivalence.

Our main interest is to count the number of realizations of generically rigid graphs, namely graphs for which almost all realizable labelings induce rigidity.

Definition 2.3. A graph $G$ is called generically realizable if for a general labeling $\lambda$ the labeled graph $(G, \lambda)$ is realizable. A graph $G$ is called generically rigid if for a general labeling $\lambda$ the labeled graph $(G, \lambda)$ is rigid.

The number of realizations can be found by solving the system of equations

$$
\left(\left(x_{u}-x_{v}\right)^{2}+\left(y_{u}-y_{v}\right)^{2}=\lambda_{u v}\right)_{\{u, v\} \in E} .
$$

Equivalently, we can study the map $r_{G}$ whose preimages correspond to the solutions of the above system

$$
r_{G}: \mathbb{C}^{2|V|} \longrightarrow \mathbb{C}^{E}, \quad\left(x_{v}, y_{v}\right)_{v \in V} \longmapsto\left(\left(x_{u}-x_{v}\right)^{2}+\left(y_{u}-y_{v}\right)^{2}\right)_{\{u, v\} \in E} .
$$

Still we get infinitely many solutions due to translations and rotations. Translations can be eliminated by moving one vertex to the origin. In order to handle rotations we perform the following transformation $x_{v} \rightarrow x_{v}+i y_{v}, y_{v} \rightarrow x_{v}-i y_{v}$. Then the above equations become

$$
\left(\left(x_{u}-x_{v}\right)\left(y_{u}-y_{v}\right)=\lambda_{u v}\right)_{\{u, v\} \in E} \cdot
$$


In this way, solutions that differ only by a rotation are the same in a suitable projective setting. If we transform the map $r_{G}$ accordingly we obtain a map whose degree is finite and gives the sought number of realizations.

In order to set up a recursive formula for the degree of the map, we want to be able to handle the two factors $\left(x_{u}-x_{v}\right)$ and $\left(y_{u}-y_{v}\right)$ independently. To do this we duplicate the graph, and for technical reasons we allow a more general class of graphs. The resulting concept is roughly speaking a pair of graphs $(G, H)$ with a bijection between their sets of edges. We identify edges by this bijection.

Definition 2.4. A bigraph is a pair of finite undirected graphs $(G, H)$ - allowing several components, multiple edges and self-loops - where $G=(V, \mathcal{E})$ and $H=(W, \mathcal{E})$. The set $\mathcal{E}$ is called the set of biedges, and there are two maps that assign to each $e \in \mathcal{E}$ the corresponding vertices in $V$ and $W$, respectively. Note that $G$ and $H$ are in general different graphs but there is a bijection between their sets of edges.

We define the Laman number $\operatorname{Lam}(B)$ of a bigraph $B$ as the degree of an associated map defined in a similar way as $r_{G}$. Moreover, we show (Proposition 2.9) that the Laman number of a graph equals the Laman number of the corresponding bigraph.

The idea for proving our recursion formula is inspired by tropical geometry: we consider the equation system over the field of Puiseux series; an algebraic relation between Puiseux series implies a piecewise linear relation between their orders. We encode these piecewise linear relations in a combinatorial data which we call bidistance. A bidistance is a pair of functions from the edges to rational numbers, which satisfies certain conditions. Using a bidistance $d$ of a bigraph $B$ we can define a new bigraph $B_{d}$ with the same number of edges. The solutions of the equations for the bigraph $B$ that correspond to the bidistance $d$ are in bijection with the solutions of the equations for $B_{d}$. Then the solutions for $B$ are partitioned by the bidistances so that we get the following formula for the Laman number:

$$
\operatorname{Lam}(B)=\sum_{d} \operatorname{Lam}\left(B_{d}\right) .
$$

From this we finally show the combinatorial recursion formula (Theorem 2.8). For doing so we prove that $\operatorname{Lam}\left(B_{d}\right)$ is either easy to compute or the product of two Laman numbers of bigraphs with fewer edges each. We need some more notation to state the theorem.

Definition 2.5. Let $B=(G, H)$ be a bigraph with biedges $\mathcal{E}$, then we say that $B$ is pseudoLaman if $\operatorname{dim}(G)+\operatorname{dim}(H)=|\mathcal{E}|+1$, where $\operatorname{dim}(G):=|V|-\mid\{$ connected components of $G\} \mid$.

It can be easily seen, that if $G$ is a Laman graph, then the bigraph $(G, G)$ is pseudo-Laman. From a given bigraph we want to construct new ones with a smaller number of edges. We introduce two constructions, a quotient and a complement, both for usual graphs and for bigraphs.

Definition 2.6. Let $G=(V, E)$ be a graph, and let $E^{\prime} \subseteq E$. We define two new graphs, denoted $G / E^{\prime}$ and $G \backslash E^{\prime}$, as follows.

$\triangleright$ Let $G^{\prime}$ be the subgraph of $G$ determined by $E^{\prime}$. Then we define $G / E^{\prime}$ to be the graph obtained as follows: its vertices are the equivalence classes of the vertices of $G$ modulo the relation dictating that two vertices $u$ and $v$ are equivalent if there exists a path in $G^{\prime}$ connecting them; its edges are determined by edges in $E \backslash E^{\prime}$. 
$\triangleright$ Let $\hat{V}$ be the set of vertices of $G$ that are endpoints of some edge not in $E^{\prime}$. Set $\hat{E}=E \backslash E^{\prime}$. Define $G \backslash E^{\prime}=(\hat{V}, \hat{E})$.

Definition 2.7. Let $B=(G, H)$ be a bigraph, where $G=(V, \mathcal{E})$ and $H=(W, \mathcal{E})$. Given $\mathcal{M} \subseteq \mathcal{E}$, we define two bigraphs ${ }^{\mathcal{M}} B=(G / \mathcal{M}, H \backslash \mathcal{M})$ and $B^{\mathcal{M}}=(G \backslash \mathcal{M}, H / \mathcal{M})$, with the same set of biedges $\mathcal{E}^{\prime}=\mathcal{E} \backslash \mathcal{M}$.

Finally, the main result can be stated.

Theorem 2.8. Let $B=(G, H)$ be a pseudo-Laman bigraph with biedges $\mathcal{E}$. Let $\bar{e}$ be a fixed biedge, then

$\triangleright$ If $G$ or $H$ has a self-loop, then $\operatorname{Lam}(B)=0$.

$\triangleright$ If both $G$ and $H$ consist of a single edge joining two different vertices, then $\operatorname{Lam}(B)=1$.

$\triangleright$ Otherwise

$$
\operatorname{Lam}(B)=\operatorname{Lam}\left({ }^{\{\bar{e}\}} B\right)+\operatorname{Lam}\left(B^{\{\bar{e}\}}\right)+\sum_{(\mathcal{M}, \mathcal{N})} \operatorname{Lam}\left(\mathcal{M}_{B}\right) \cdot \operatorname{Lam}\left(B^{\mathcal{N}}\right),
$$

where each pair $(\mathcal{M}, \mathcal{N}) \subseteq \mathcal{E}^{2}$ satisfies:

$$
\begin{aligned}
& \diamond \mathcal{M} \cup \mathcal{N}=\mathcal{E} ; \\
& \diamond \mathcal{M} \cap \mathcal{N}=\{\bar{e}\} ; \\
& \diamond|\mathcal{M}| \geq 2 \text { and }|\mathcal{N}| \geq 2 ; \\
& \diamond \text { both }{ }^{\mathcal{M}} B \text { and } B^{\mathcal{N}} \text { are pseudo-Laman. }
\end{aligned}
$$

Furthermore, we show the following proposition in [2], which completes the recursive algorithm for computing Laman numbers.

Proposition 2.9. The number of realizations of a Laman graph $G$ is equal to the Laman number of the bigraph $(G, G)$.

\section{Conclusion}

Using the recursion formula from Theorem 2.8 we were able to compute Laman numbers for all Laman graphs up to 12 vertices. Furthermore, we computed Laman numbers for single graphs up to 18 vertices, which was out of reach with the previous methods. For further details and proofs we refer to the aforementioned paper [2]. Additional information including implementations in Mathematica and in C++ can be found at www.koutschan.de/data/laman/.

\section{References}

[1] Borcea, C. and I. Streinu, The number of embeddings of minimally rigid graphs, Discrete \& Computational Geometry 31 (2004), pp. 287-303.

[2] Capco, J., M. Gallet, G. Grasegger, C. Koutschan, N. Lubbes and J. Schicho, The number of realizations of a Laman graph, Technical Report 2017-03, Johann Radon Institute for Computational and Applied Mathematics (2017), arXiv:1701.05500. 
[3] Emiris, I., E. Tsigaridas and A. Varvitsiotis, Mixed volume and distance geometry techniques for counting Euclidean embeddings of rigid graphs, in: Distance geometry, Springer, New York, 2013 pp. 23-45.

[4] Graver, J., B. Servatius and H. Servatius, "Combinatorial rigidity," American Mathematical Society, Providence, RI, 1993.

[5] Jackson, B. and J. Owen, The number of equivalent realisations of a rigid graph, Technical Report 1204.1228, arXiv (2012).

[6] Laman, G., On graphs and rigidity of plane skeletal structures, Journal of Engineering Mathematics 4 (1970), pp. 331-340.

[7] Steffens, R. and T. Theobald, Mixed volume techniques for embeddings of Laman graphs, Computational Geometry 43 (2010), pp. 84-93. 